\title{
Environmental enrichment alleviates chronic pain in rats following a spared nerve injury to induce neuropathic pain. A preliminary study
}

This article was published in the following Dove Press journal: Veterinary Medicine: Research and Reports

\author{
Madeleine Parent-Vachon \\ Pascal Vachon \\ Department of Veterinary \\ Biomedicine, Faculty of Veterinary \\ Medicine, University of Montreal, \\ Saint-Hyacinthe, QC J2S 2M2, Canada
}

Correspondence: Pascal Vachon Department of Veterinary Biomedicine, Faculty of Veterinary Medicine, University of Montréal, 3200 Sicotte, SaintHyacinthe, QC J2S 2M2, Canada Tel +l 450773852 I ext 8294 Email pascal.vachon@umontreal.ca
Background: In mice, chronic pain can be alleviated with enriched environments (EEs). The purpose of this preliminary study is to investigate whether pain behaviors in rats with peripheral neuropathy would be altered when keeping these animals in either 1) standard laboratory cages or in 2) a significantly EE.

Methods: Two groups of rats ( $n=8$ /group) underwent a spare nerve injury surgery of the right hind leg; one group $(n=8)$ was returned to standard ventilated cages ( 2 rats/cage), the other $(n=8)$ placed in an EE ( 8 rats/ferret cage with toys). A third group $(n=8)$ underwent a sham surgery and was used as control. These animals were returned to standard ventilated cages ( 2 rats per cage). Spare nerve injury surgery consisted of ligation/transection of the tibial and common peroneal branches of the sciatic nerve of the right leg only. Von Frey Filaments were applied to test mechanical sensitivity of both hind paws.

Results: The right paw of nerve-injured animals was hypersensitive to mechanical stimuli at 2, 4, and 8 weeks following the surgery; however, animals in the EE conditions showed significantly $(P<0.05)$ less mechanical sensitivity than rats left in the standard caging environment (2, 4, and 8 weeks postsurgery: standard environment $2.8 \pm 0.5,2.8 \pm 0.7$, and $2.6 \pm 0.4$ and $\mathrm{EE}$ $4.7 \pm 0.6,5.8 \pm 0.5$, and $5.5 \pm 0.7)$. Sham animals were unaffected by the surgery.

Conclusion: Environmental enrichment alleviated mechanically induced chronic pain in a spared nerve injury rat model of neuropathic pain. Findings also suggest that environmental enrichment, as a method to alleviate pain, may be species-specific, motor behaviors being a very important parameter when considering pain modulation.

Keywords: environmental enrichment, spare nerve injury, neuropathy, chronic pain, rats

\section{Introduction}

Environmental enrichment used to increase animal welfare is gaining interest in laboratory animal medicine, and is important for alleviation of chronic pain. Studies in both animals ${ }^{1-8}$ and humans ${ }^{9,10}$ looking at environmental effects on pain recovery following surgery show a significant alleviation of pain with environmental enrichment.

Rodents living in enriched environments (EEs) show less pain behaviors following a peripheral nerve injury than those living in a standardized laboratory cage environment. ${ }^{5-8}$ However, in these studies, animals were kept for a short period of time $(<1$ month) in the EE from the start of the study. In mice, it has recently been shown that pain can be alleviated with environmental enrichment well after the establishment of chronic pain. ${ }^{3,4}$ In this preliminary study, we investigated whether pain following an induced peripheral neuropathy in rats would be altered when keeping these animals in a significantly EE compared to standard laboratory cages over a 
period of 2 months. Our previous experiments ${ }^{3,4}$ show that pain can be alleviated in mice with environmental enrichment, whereas a minimally EE in rats showed no effect with regard to the alleviation of chronic pain. ${ }^{11}$ In the present experiment, environmental enrichment was significantly modified compared to laboratory animal research standards by increasing cage size and number of "toys," as well as increased opportunities for social interactions, to assess whether these modifications would be sufficient to alleviate chronic pain in rats.

\section{Materials and methods}

\section{Animals and husbandry}

Twenty-four Sprague Dawley rats (CRL:CD[SD]; Charles River, Saint-Constant, QC, Canada) weighing between 225 and $250 \mathrm{~g}$ were used for this study. Following their arrival, they were kept in a standard laboratory animal environment (fresh filtered air, 15 changes/hour; temperature, $21^{\circ} \mathrm{C} \pm 2{ }^{\circ} \mathrm{C}$; humidity $50 \% \pm 20 \%$; and light/dark cycle, $12: 12$ hours). Rats were housed (groups of 2) on hardwood bedding (Beta chip, Northeastern Products, Warrensburg, NY, USA) in ventilated cages (Green Line IVC Sealsafe Plus, Tecniplast, West Chester, PA, USA) with only black PVC tubing for environmental enrichment. Following their arrival, and a 1 week acclimation period, rats were then acclimated daily to the von Frey equipment for 1 week and underwent surgery the following week.

Following the spare nerve injury (SNI) surgery, one group of rats $(n=8$; SNI-SE) was returned to the ventilated cages ( 2 rats/cage) and another group $(n=8 ;$ SNI-EE) placed in groups of eight in ferret cage (Ferret Home cage 485, Pet Products, Chicago, IL, USA; dimensions: 31 inches long, 20 inches wide, and 41.5 inches high with 7/8-inch wire spacing) with toys (colored balls, paper, PVC tubing, plastic spirals). One group underwent a sham surgery ( $n=8$; Sham-SE), and these animals served as negative controls. Following surgery they were returned to the ventilated cages ( 2 rats per cage) in a standard environment. All rats received tap water and a standard laboratory rodent diet (Charles River Rodent Chow 5075, Charles River) ad libitum. The experimental protocol was approved by the University of Montreal institutional animal care committee prior to animal use, in accordance with the guidelines of the Canadian Council on Animal Care. ${ }^{12}$

\section{Surgical procedures}

SNI surgery consisted of ligation and transection of the tibial and common peroneal branches of the sciatic nerve of the right leg only, while sparing the sural nerve. ${ }^{13}$ Surgeries were performed under general anesthesia ( $2 \%$ isoflurane in
$1 \mathrm{~L} \mathrm{O}_{2} / \mathrm{min}$ ). The tibial and common peroneal nerves were ligated with silk suture (Ethicon 6/0) and cut. The skin was closed with $6 / 0$ silk, and topical antiseptic polysporin cream (bacitracin/polymyxin) was applied. Sham surgery involved exposing the nerve without damaging it.

\section{Behavioral evaluations}

All behavioral evaluations were performed in a designated rodent behavioral testing room with dimed lights. The threshold to withdraw was calculated as previously described. ${ }^{14}$ Calibrated von Frey filaments (Stoelting Co., Wood Dale, IL, USA) were applied for 4 seconds, or until paw withdrawal, to test mechanical sensitivity of both limbs. The applied force ranged from 1 to $26 \mathrm{~g}$, corresponding to filament sizes 4.08-5.46. For each animal, the actual filaments used within the aforementioned series were determined based on the lowest filament to evoke a positive response using the up-down method. Rats were acclimated to the experimental setup 15 minutes prior to testing. The mechanical sensitivity was assessed on the lateral plantar surface of both hind paws. Rats were randomly tested, mixing all three experimental groups. The person testing the animals was blinded to all experimental conditions.

\section{Statistical analysis}

Statistical analyses and graphs were performed using GraphPad Prism 4.0 (GraphPad Software, La Jolla, CA, USA). The time course for sensory, motor, and affective measures was analyzed by two-way repeated-measures analysis of variance (RM-ANOVA) followed by the Bonferroni post hoc test for multiple comparisons. All data are expressed and plotted as mean \pm standard error of the mean. $P<0.05$ was considered statistically significant. If a significant effect of environment was observed in the absence of a time effect or an environment $\times$ time interaction, one-way RM-ANOVA was used to determine if a time effect existed within each group (ie, neuropathic enriched).

\section{Results}

Neuropathic rats with environmental enrichment had significantly reduced hypersensitivity to mechanical stimuli (Figure 1 ; enriched vs impoverished, two-way RM-ANOVA, $F(1,18)$ $=4.9, P<0.05)$ compared to animals placed in standard cages. This decreased hypersensitivity was observed at 2, 4, and 8 weeks following the surgery. No significant changes of sensitivity to mechanical stimuli were observed in control rats that underwent a sham surgery. For all three experimental groups, the nonoperated paw sensitivities were between $22.7 \pm 0.5$ and $24.6 \pm 0.6 \mathrm{~g}$ (not shown). 


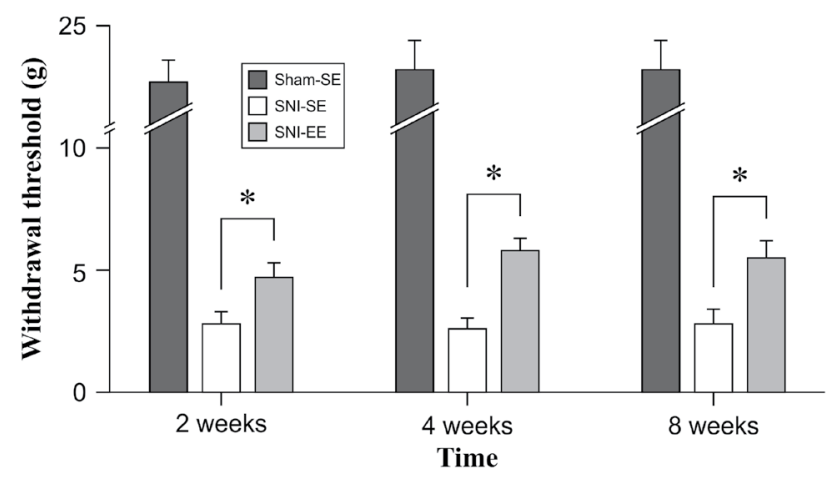

Figure I Mean $( \pm$ SEM) mechanical sensitivities evaluated with von Frey filaments in different experimental groups (SNI-EE and SNI-SE) and Sham-SE evaluated at 2, 4, and 8 weeks following the surgery.

Notes: Data presented is the withdrawal threshold (in grams) corresponding to the filament size at which the animals reacted in the right hind paw only. SNI animals in both environmental conditions were sensitized however rats in the EE show less mechanical sensitivity than the $\mathrm{SNI}$ animals in the standard caging environment $(* P<0.05)$.

Abbreviations: SEM, standard error of the mean; Sham-SE, sham-operated anima in a standard caging environment; SNI-EE, spared nerve injury rats with environmental enrichment; SNI-SE, spared nerve injury rats in ventilated cages.

\section{Discussion}

Our results show that environmental enrichment has a statistically significant but moderate impact on pain hypersensitivity in a rat model of neuropathic pain. Environmental enrichment significantly decreased pain thresholds to mechanicalinduced pain in rats, an effect that has previously been shown in mice. ${ }^{4}$ Interestingly in mice, enrichment cages decreased anxiety and increased exploration; however, in rats, increasing the cage size without adding toys did not modify pain behavior, but animals were found to be less anxious with an increase in exploratory behavior. ${ }^{11}$ Importantly, even if the ferret cage environment alleviated pain, rats were still sensitive to mechanical stimuli and would be considered neuropathic, as was observed in mice, suggesting that environment enrichment in itself is insufficient to cure pain. Altogether our findings suggest that significantly increasing rat cage size, providing more environmental enrichment, and favoring social interactions can alleviate neuropathic pain. Since this is a preliminary study, no attempt was made to separate out these different parameters to assess the importance of each in alleviating pain. Many animal models used in research (eg, cancer, musculoskeletal problems, etc.) will suffer from chronic pain, and these animals would benefit of a more appropriate environment to alleviate pain without medications.

One of the main components of the EE in the mouse study was a running wheel, ${ }^{4}$ and exercise is known to decrease pain symptoms and improve motor function in chronic pain models. This is also an important component of the ferret cage, since rats had a significantly increased opportunity for exploratory behavior, moving freely on different levels of the cage. However, the ferret cage may not have been challenging enough, in terms of exercise, and a running wheel may be more appropriate to increase exercise. However, rats are known to be physically much less active than mice.

Stagg et a $1^{15}$ demonstrated that thermal hyperalgesia in a rat model of neuropathic pain is reversed with exercise. Interestingly, reversal of sensory hypersensitivity was seen even when exercise was initiated 4 weeks after spinal nerve ligation, and returned 5 days after discontinuing exercise. Exercise appears sufficient to have a significant alleviating effect on neuropathic pain symptoms, and clinical studies in humans suggest that exercise decreases pain symptoms in chronic pain patients. ${ }^{16-18}$ However in our study the results with mechanical stimuli did not show the same results.

\section{Conclusion}

Environmental enrichment decreased mechanically induced chronic pain in an SNI rat model of neuropathic pain. Animal models used in research (eg, cancer, musculoskeletal problems, etc) may suffer from chronic pain, and so a more appropriate environment that would alleviate pain without medications, which may interfere with research findings, is essential.

\section{Acknowledgment}

This study was funded by the Laboratory Animal Research Fund (Faculty of Veterinary Medicine, University of Montreal).

\section{Disclosure}

Madeleine Parent-Vachon, daughter of Pascal Vachon, is admitted in a Master's program of Veterinary Biomedicine at the University of Montreal. The Department of Veterinary Medicine, the Vice-Dean of student affairs of the Faculty of Veterinary Medicine, and the Faculty of Graduate Affairs have all accepted the master's project and the admission of Madeleine Parent-Vachon. The authors report no other conflicts of interest in this work.

\section{References}

1. Lankhorst AJ, Ter Laak MP, van Laar TJ, et al. Effects of enriched housing on functional recovery after spinal cord contusive injury in the adult rat. J Neurotrauma. 2001;18(2):203-215.

2. Berrocal Y, Pearse DD, Singh A, et al. Social and environmental enrichment improves sensory and motor recovery after severe contusive spinal cord injury in the rat. J Neurotrauma. 2007;24(11):1761-1772. 
3. Tajerian M, Alvarado S, Millecamps M, et al. Peripheral nerve injury is associated with chronic, reversible changes in global DNA methylation in the mouse prefrontal cortex. PLOS ONE. 2013;8(1):e55259.

4. Vachon P, Millecamps M, Low L, et al. Alleviation of chronic neuropathic pain by environmental enrichment in mice well after the establishment of chronic pain. Behav Brain Funct. 2013;9:22.

5. Gabriel AF, Marcus MA, Honig WM, Helgers N, Joosten EA. Environmental housing affects the duration of mechanical allodynia and the spinal astroglial activation in a rat model of chronic inflammatory pain. Brain Res. 2009;1276:83-90.

6. Gabriel AF, Paoletti G, della Seta D, et al. Enriched environment and the recovery from inflammatory pain: social versus physical aspects and their interaction. Behav Brain Res. 2010;208(1):90-95.

7. Gabriel AF, Marcus MA, Honig WM, Joosten EA. Preoperative housing in an enriched environment significantly reduces the duration of post-operative pain in a rat model of knee inflammation. Neurosci Lett. 2010;469(2):219-223

8. Tall JM. Housing supplementation decreases the magnitude of inflammation-induced nociception in rats. Behav Brain Res. 2009;197(1):230-233.

9. Smith MA, Bryant PA, Mcclean JM. Social and environmental enrichment enhances sensitivity to the effects of kappa opioids: studies on antinociception, diuresis and conditioned place preference. Pharmacol Biochem Behav. 2003;76(1):93-101.

10. Ulrich RS. View through a window may influence recovery from surgery. Science. 1984;224(4647):420-421.
11. Vachon P. Double decker enrichment cages have no effect on long term nociception in neuropathic rats but increases exploration while decreasing anxiety-like behaviors. Scand J Lab Anim Sci. 2014;40.

12. Canadian Council on Animal Care. Guide to the Care and Use of Experimental Animals. Ottawa, ON: Canadian Council on Animal Care; 1993.

13. Decosterd I, Woolf CJ. Spared nerve injury: an animal model of persistent peripheral neuropathic pain. Pain. 2000;87(2):149-158.

14. Chaplan SR, Bach FW, Pogrel JW, Chung JM, Yaksh TL. Quantitative assessment of tactile allodynia in the rat paw. J Neurosci Methods. 1994;53(1):55-63.

15. Stagg NJ, Mata HP, Ibrahim MM, et al. Regular exercise reverses sensory hypersensitivity in a rat neuropathic pain model: role of endogenous opioids. Anesthesiology. 2011;114(4):940-948.

16. Gowans SE, Dehueck A, Voss S, Silaj A, Abbey SE. Six-month and one-year followup of 23 weeks of aerobic exercise for individuals with fibromyalgia. Arthritis Rheum. 2004;51(6):890-898.

17. Robb KA, Williams JE, Duvivier V, Newham DJ. A pain management program for chronic cancer-treatment-related pain: a preliminary study. J Pain. 2006;7(2):82-90.

18. Chatzitheodorou D, Kabitsis C, Malliou P, Mougios V. A pilot study of the effects of high-intensity aerobic exercise versus passive interventions on pain, disability, psychological strain, and serum cortisol concentrations in people with chronic low back pain. Phys Ther. 2007;87(3):304-312.
Veterinary Medicine: Research and Reports

\section{Publish your work in this journal}

Veterinary Medicine: Research and Reports is an international, peer-reviewed, open access journal publishing original research, case reports, editorials, reviews and commentaries on all areas of veterinary medicine. The manuscript management system is completely online and includes a very quick and fair peer-review system.
Dovepress

Visit http://www.dovepress.com/testimonials.php to read real quotes from published authors. 